\title{
Amenorrea Secundaria Estudio y Análisis de 124 Casos
}

\author{
Dres. Jorge Mario Mejía R., Hernán Alberto Giraldo E., \\ Guillermo Henao C. y Fabio Sánchez E.
}

Grupo de Ginecología Endocrina y Reproducción Humana.

Departamento de Obstetricia y Ginecología

Centro de Investigaciones Médicas

Facultad de Medicina

Universidad de Antioquia

Hospital Universitario San Vicente de Paúl

\section{INTRODUCCION}

Lo más importante para la paciente con Amenorrea Secundaria es tener de nuevo sus menstruaciones, pero para el clínico lo fundamental debe ser identificar claramente la etiología, que puede variar desde una disfunción del eje hipotálamo-hipófisis-ovario hasta una causa orgánica que repercuta sobre su fertilidad, o lo que es más grave, comprometer seriamente órganos y aún la misma vida de la paciente (10).

Siempre ha sido un reto para el médico el enfoque de la Amenorrea Secunda ria y se han propuesto varios esquemas de estudio $(1,2,5,6,8,9,10,13,15)$, tratando de identificar en el menor tiempo y con los mínimos costos el factor etiológico para poder dar la terapia ade-
REIMPRESOS:

Jorge Mario Mejía Restrepo

Departamento de Obstetricia y Ginecología Hospital Universitario San Vicente de Paúl Medellín - Colombia - Sur América

cuada a cada caso y no conformarse, como la paciente, en tratar el síntoma de falta de menstruación.

Es el propósito de este trabajo señalar nuestra experiencia clínica en el diagnóstico de la Amenorrea Secundaria, empleando un esquema de estudio en el cual no se utiliza la clásica prueba de supresión con progesterona $(8,11)$ y analizar varios datos encontrados en estas pacientes.

\section{Material y Métodos}

Se revisaron las historias clínicas de las pacientes que consultaron al Grupo de Ginecología Endocrina y Reproducción Humana del Hospital Universitario San Vicente de Paúl (Universidad de Antioquia), por Amenorrea Secundaria 
de duración mayor de tres meses, durante un período de 4 años (1976-1978). Durante este lapso se llevó la siguiente metodología con todas las pacientes: Historia clínica y examen físico completo, exclusión de embarazo, biopsia de endometrio, citología funcional y determinación de prolactina. De acuerdo con los resultados de los exámenes anteriores a las pacientes se les ordenaba o no, uno o varios de los siguientes exámenes: Hormona folículo estimulante (FSH), hormona luteinizante (LH), 17 hidroxiesteroides, 17 ketosteroides, hormona tiroestimulante (TSH), hormonas tiroideas (T3 y T4), histerosalpingografía, tomografía de silla turca y otro que se necesitara para el diagnóstico, (Tabla No. 1).
No se utilizó en el estudio, la clásica prueba de Supresión con Progesterona y ocasionalmente se requirió la laparoscopia diagnóstica. Se agruparon los diagnósticos de acuerdo con la clasificación de la Amenorrea Secundaria establecida por la Organización Mundial de la Salud (17) que la divide en VII Grupos: (Tabla No. 9).

\section{Resultados}

Entre 1.250 pacientes que consultaron al programa durante cuatro años (1976-1979), 124 lo hicieron por Amenorrea Secundaria mayor de tres meses, número que corresponde al $9.9 \%$ de laspacientes, (Tabla No. 2).

Tabla No. 1

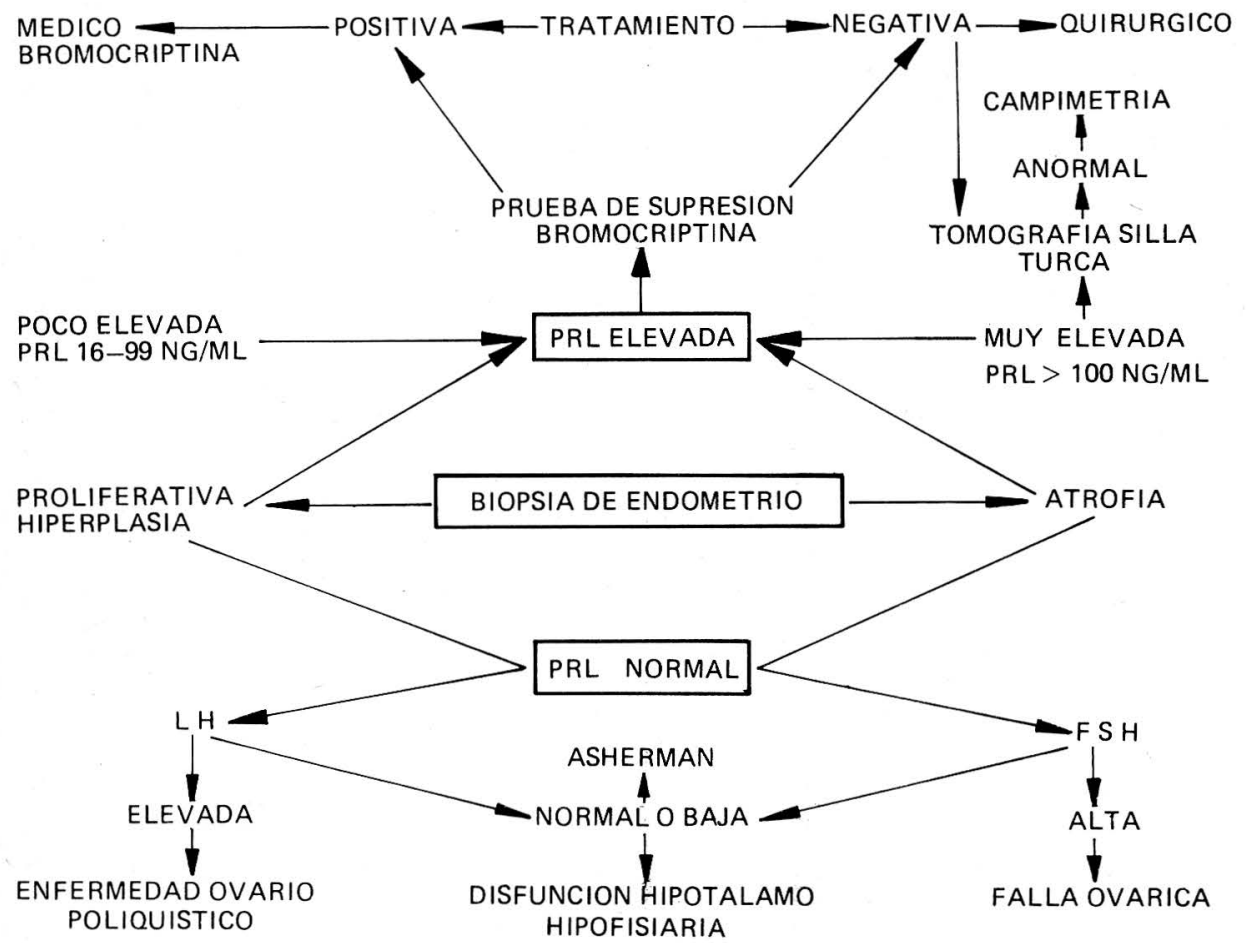

H.U.S.V. DE P. 
Tabla No. 9

\section{CLASIFICACION DE \\ LA AMENORREA SECUNDARIA ORGANIZACION MUNDIAL DE LA SALUD}

Falla hipotálamo - hipofisiaria sin tumor

II Disfunción hipotálamo - hipofisiaria con PRL NORM.

III Falla Ovárica

IV Causa orgánica adquirida útero y vagina

$\checkmark$ Hiperprolactinemia con tumor

VI Hiperprolactinemia sin tumor

VII Tumor hipotálamo - hipófisis con PRL normal

SIMPOSIUM DE LA OMS: REGULACION DE FERTILIDAD HUMANA - MOSCU 1.976

Tabla No. 2

FRECUENCIA DE AMENORREA

PATOLOGICA EN

1.250 PACIENTES

HUSVP - U. de A.

1.976-1.979

\begin{tabular}{lrr}
\hline Amenorrea & No. & Fr. O/o \\
\hline Primaria & 54 & 4.3 \\
Secundaria & 124 & 9.9 \\
\hline Total & 178 & 14.1
\end{tabular}

El Grupo de edad en el cual se presentó con mayor frecuencia la Amenorrea Secundaria, fue comprendido entre 20 y 24 años con 41 pacientes $(33 \%)$, (Tabla No. 4).

En cuanto a la duración de la Aménorrea, en 79 pacientes $\left(63.7^{\circ}, \mathrm{o}\right)$ fue mayor de seis meses $y$ el resto, 45 pacientes $(36.3 \%)$ entre tres $y$ seis meses (Tabla No. 8).

La Amenorrea fue precedida por períodos de oligomenorrea en 41 pacientes
$(66.1 \%)$ y en 21 casos $(33.9 \%)$ seestableció de manera súbita, (Tabla No. 7).

Tabla No. 4

DISTRIBUCION DE LA EDAD

EN 124 PACIENTES CON

AMENORREA SECUNDARIA

HUSVP-U. de A.

1.976-1.979

\begin{tabular}{ccc}
\hline Años & No. & FrO/o \\
\hline$<19$ & 18 & 14.5 \\
$20-24$ & 41 & 33.0 \\
$25-29$ & 30 & 24.2 \\
$30-34$ & 22 & 17.7 \\
35 o Más & 13 & 10.4 \\
\hline Total & 124 & 100.0 \\
\hline
\end{tabular}

Tabla 8

TIEMPO TRANSCURRIDO ENTRE LA INICIACION DE LA AMENORREA $Y$ LA CONSULTA HUSVP-U. de A. 1.976-1.979

Tiempo en Meses

No.

$0 / 0$

\begin{tabular}{rrr}
\hline $3-6$ & 43 & 34.7 \\
$7-12$ & 53 & 42.7 \\
$>13$ & 28 & 22.6 \\
\hline Total & 124 & 100.0
\end{tabular}

Los ciclos menstruales iniciales de 94 pacientes fueron regulares en 52 de ellas $(55.3 \%)$ e irregulares en $42(44.7 \%)$, (Tabla No. 6). 
Tabla 7

FORMA DE INSTALACION DE LA AMENORREA SECUNDARIA HUSVP-U. de A. 1.976-1.979

\begin{tabular}{lll}
\hline Instalación & No. & \%o \\
\hline Progresiva & 41 & 66.1 \\
Súbita & 21 & 33.9 \\
\hline Total & 62 & 100.0 \\
\hline
\end{tabular}

Tabla 6

CICLOS MENSTRUALES INICIALES EN 94 PACIENTES CON AMENORREA SECUNDARIA HUSVP- U. de A. 1.976-1.979

\begin{tabular}{lcr}
\hline $\begin{array}{l}\text { Ciclos Menstruales } \\
\text { Iniciales }\end{array}$ & No. & Fr. \% \\
\hline Regulares & 52 & 55.3 \\
Irregulares & 42 & 44.7 \\
\hline Total & 94 & 100.0 \\
\hline
\end{tabular}

Se encontró que 70 pacientes $(56.9 \%)$ eran casadas y el resto $(43.1 \%)$ solteras, (Tabla No. 5).

De las 124 pacientes, $41(33.1 \%)$ presentaron una Amenorrea Galactorrea, (Tabla No. 12).

En el presente estudio sólo se pudo llegar a un diagnóstico etiológico y efectuar un tratamiento adecuado en 71 pacientes $(57 \%)$; en el resto de ellas no fue posible debido a la deserción antes de llegar a un diagnóstico etiológico.
Tabla No. 5

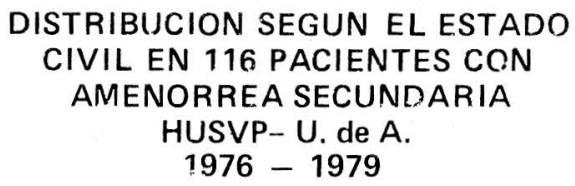

\begin{tabular}{lcc}
\hline Estado Civil & No. Casos & Fr. \% \\
\hline Casadas & 66 & 56.9 \\
Solteras & 50 & 43.1 \\
\hline Total & 116 & 100.0
\end{tabular}

Tabla 12

\section{FRECUENCIA DE GALACTORREA EN 124 PACIENTES CON AMENORREA SECUNDARIA HUSVP $-U$. de A. 1.976-1.979}

\begin{tabular}{ccc}
\hline Galactorrea & No. & \% \\
\hline Si & 41 & 33.06 \\
No & 83 & 66.93 \\
\cline { 2 - 3 } Total & 124 & 100.00 \\
\hline
\end{tabular}

Tabla No. 3

FRECUENCIA DE AMENORREA SECUNDARIA EN 178 PACIENTES CON AMENORREA PATOLOGICA HUSVP-U. de A. 1.976-1.979

\begin{tabular}{lcc}
\hline Amenorrea & No. Casos & \% \\
\hline Secundaria & 124 & 69.7 \\
Primaria & 54 & 30.3 \\
\hline Total & 178 & 100.0 \\
\hline
\end{tabular}


Tabla No. 10

ETIOLOGIA DE LA AMENORREA SECUNDARIA EN 71 PACIENTES

HUSVP-U. de A.

1.976-1.979

\begin{tabular}{|c|c|c|c|}
\hline Grupo & & No. & $\%$ \\
\hline \multirow[t]{3}{*}{ IV } & $\begin{array}{l}\text { Causa orgánica adquirida } \\
\text { de útero y vagina }\end{array}$ & 7 & 9.9 \\
\hline & Asherman & 6 & 8.5 \\
\hline & Bridas vaginales & 1 & 1.4 \\
\hline V & $\begin{array}{l}\text { Hiperprolactinemia con } \\
\text { tumor }\end{array}$ & 7 & 9.9 \\
\hline \multirow[t]{5}{*}{ VI } & Hiperprolactinemia $\sin$ & & \\
\hline & tumor & 13 & 18.3 \\
\hline & Idiopática & 8 & 11.3 \\
\hline & Postpildora & 4 & 5.6 \\
\hline & post-parto & $i$ & 1.4 \\
\hline
\end{tabular}

Tabla 11

ETIOLOGIA DE LA AMENORREA

SECUNDARIA EN 71 PACIENTES

HUSVP-U. de A.

1.976-1.979

\begin{tabular}{|c|c|c|c|}
\hline Grupo & & No. & $\%$ \\
\hline 1 & $\begin{array}{l}\text { Falla hipotálamo hipófisis } \\
\text { sin tumor }\end{array}$ & & \\
\hline \multirow{7}{*}{11} & Sindrome de Sheehan & 3 & 4.2 \\
\hline & $\begin{array}{l}\text { Disfunción hipotálamo hi- } \\
\text { pófisis con prolactina nor- }\end{array}$ & 37 & 521 \\
\hline & Ovario androgénico & 27 & 38.0 \\
\hline & Postpíldora & 5 & 7.1 \\
\hline & Disminución de peso & 3 & 4.2 \\
\hline & Post parto & 1 & 1.4 \\
\hline & Pseudóciesis & 1 & 1.4 \\
\hline III & $\begin{array}{l}\text { Falla ovárica } \\
\text { Menopausia precoz }\end{array}$ & 4 & 5.6 \\
\hline
\end{tabular}

De acuerdo con la clasificación adoptada por la Organización Mundial de la Salud, la cual divide la Amenorrea Se- cundaria en VII Grupos (17), nuestras 71 pacientes se pueden distribuir así:

En el Grupo I. FALLA HIPOTALAMO HIPOFISIARIA tres pacientes $(4.2 \%)$, las cuales correspondían al Síndrome de Sheehan. En el Grupo II. DISFUNCION HIPOTALAMO - HIPOFISIARIA CON PROLACTINA NOR$M A L$, le correspondió el mayor número de pacientes, $37(52.1 \%)$ y la entidad, más frecuentemente responsable de esta disfunción fue la enfermedad de ovario androgénico con 27 pacientes $(38.0 \%)$,

\section{Tabla 13}

\section{NIVELES DE PROLACTINA EN 71 PACIENTES CON AMENORREA SECUNDARIA HUSVP-U. de A.}

\subsection{6-1.979}

\begin{tabular}{llr}
\hline Prolactina & No. & Fr. $\%$ \\
\hline Normal & 51 & 71.9 \\
Elevada & 20 & 28.1 \\
\hline Total & 71 & 100.0 \\
\hline
\end{tabular}

Tabla 14

\begin{tabular}{|c|c|c|}
\hline \multicolumn{3}{|c|}{$\begin{array}{c}\text { NIVELES DE PROLACTINA } \\
\text { EN } 26 \text { PACIENTES CON } \\
\text { AMENORREA - GALACTORREA } \\
\text { HUSVP-U. de A. } \\
1.976-1.979\end{array}$} \\
\hline Prolactina & No. & $\mathrm{Fr} . \mathrm{O} / \mathrm{O}$ \\
\hline Normal & 12 & 46.2 \\
\hline Elevada & 14 & 53.8 \\
\hline Total & 26 & 100.0 \\
\hline
\end{tabular}


tres pacientes $(4.2 \%)$ desencadenada por la disminución de peso, cinco pacientes $(7.1 \%)$ posterior a la suspensión de la ingesta de anticonceptivos orales ("postpildora") y una paciente $(1.4 \%$ ) postparto. Al Grupo III. FALLA OVARICA se incluyeron cuatro pacientes $(5.6 \%)$ con menopausia precoz. En el Grupo IV. CAUSA ORGANICA DE UTERO Y VAGINA fueron siete pacientes $(9.9 \%)$, seis de ellas tenían un Síndrome de Asherman y una con bridas vaginales. Al Grupo V. HIPERPROLACTINEMIA CON TUMOR le correspondieron siete pacientes $(9.9 \%)$ con microadenomas de hipófisis. En el Grupo VI. HIPERPROLACTINEMIA SIN TUMOR, 13 pacientes $(18.3 \%)$, de las cuales ocho $(11.3 \%$ eran idiopáticas, cuatro $(5.6 \%)$ postpildora y una paciente $(1.4 \%)$ postparto. Del Grupo VII. TUMOR HIPOTALAMO HIPOFISIS CON PROLACTINA NORMAL no encontramos ninguna paciente, (Tabla No. 10 y No. 11).

\section{Discusión}

Aunque son varias las clasificaciones para la Amenorrea Secundaria (1, 2, 5, $6,8,9,10)$, nos parece que la adoptada por la Organización Mundial de la Salud (17) no sólo permite identificar el nivel de las alteraciones, sino establecer una metodología para el diagnóstico, elaborar un pronóstico de la entidad y enfocar el tratamiento de la Amenorrea ya sea para regularizar los ciclos o cuando la paciente desee quedar en embarazo.

Las principales ventajas que tiene el tomar de una vez la biopsia de endometrio después de descartado el embarazo y no usar la prueba de supresión con progesterona son las siguientes:

1. Ahorra tiempo en el estudio principalmente en las pacientes que tienen la prueba de progesterona negativa por una causa orgánica de útero que se sospecha en la toma de la biopsia y no hay que esperar que sea negativa también la prueba de estrógenos más progesterona para continuar el estudio.

2. Es mucho más confiable el informe de patología para saber que niveles de estrógenos tiene la paciente ya que muchas veces pacientes con hiperplasias atípicas no sangran con la primera prueba de supresión, (16).

3. Al presentar sangrado de supresión la paciente se siente muy tranquila $y$ a veces abandona otros estudios y algunos médicos también se conforman con ésto sin profundizar en el diagnóstico, pasándose muchas veces hiperprolactinemias moderadas.

Adoptando el esquema de biopsia de endometrio y determinación de prolactina en todas las pacientes con Amenorrea tengan o no galactorrea, permite clasificar rápidamente a la paciente en los distintos grupos adoptados por la OMS: (Tabla No. 10, 11) si la prolactina es normal y la biopsia de endometrio proliferativa o hiperplásica clasifica a estas pacientes en el Grupo II y es por la historia clínica (ejemplo: postpildora, postparto), o el exámen físico (ejemplo: disminución de peso) y una LH/FSH disociada (ejemplo: ovario androgénico) que se hace el diagnóstico etiológico. (Tabla No. 1).

Si la biopsia de endometrio es atrófica y la prolactina es normal, estas pacientes se clasifican en el Grupo I ó III de acuerdo al resultado de la FSH, si es alta se trata de una falla ovárica y si es baja una falla hipotálamo hipófisis.

Las pacientes con hiperprolactinemia (Grupos V y VI), pueden tener la biopsia atrófica o proliferativa dependiendo de los valores de prolactina, y es mediante una tomografía de silla turca que sabe- 
mos qué pacientes presentan o no adenomas.

Como ya dijimos, al tratar de tomar la biopsia de endometrio, podemos sospechar que se trata de un factor uterino, confirmándose el diagnóstico de Síndrome de Asherman con la toma de una histerosalpingografía.

Al analizar los resultados se encuentra que la Amenorrea Secundaria en una consulta especializada de Endocrinología y Esterilidad es casi el $10 \%$ de las pacientes y el $70 \%$ de las Amenorreas $\mathrm{Pa}$ tológicas que está de acuerdo con los informes de otros autores $(9,12)$. (Cuadros Nos. 2 y 3 ).

La mayoría de las pacientes $(63.7 \%)$ tenían una Amenorrea mayor de seis meses, reflejándose una patología más severa como son los Síndromes de Sheehan, Asherman, todas las pacientes con hiperprolactinemias, y las fallas ováricas; por el contrario, la mayoría de las pacientes con Amenorrea entre tres a seis meses correspondieron a una disfunción hipotálamo hipófisis con prolactina normal (ovario androgénico) que son las de mejor pronóstico desde el punto de vista de la fertilidad.

La mayor frecuencia de Amenorrea Secundaria en los grupos de edad de 20 a 29 años, se puede explicar por ser en esta edad donde se presenta el ovario androgénico que fue la causa más común de Amenorrea Secundaria $(38 \%$ ) (Cuadro No. 10) (5).

De las 71 pacientes, $20(28.1 \%)$ tienen una hiperprolactinemia (Tabla No. 13) y si tomamos las 26 pacientes con Amenorrea Galactorrea $(36.6 \%)$, más de la mitad $(53.8 \%)$ presentan, hiperprolactinemia (Tabla No. 14), por lo anterior se considera adecuado solicitar una determinación de prolactina a todas las pacientes con el diagnóstico de
Amenorrea tengan o no Galactorrea, antes de tomar una conducta terapeutica.

El $70 \%$ de las pacientes con hiperprolactinemia tienen una Amenorrea Galactorrea, dato de acuerdo con otros autores $(3,4)$; la frecuencia de microadenomas fue del $9,9 \%$ del total de pacientes con Amenorrea y del $35 \%$ de las que tienen hiperprolactinemia, dato un poco más alto que el reportado en otros informes de la literatura $(7,14)$.

Llama la atención el alto porcentaje de Síndrome de Asherman $8.5 \%$, posiblemente relacionado con el manejo obstétrico no especializado de estas pacientes en nuestro medio (5).

\section{Bibliografía}

1. SHERMAN R.P., FRASER I.S.: Impact of New Diagnostic Methods on the Differential Diagnosis and Treatment of Secondary Amenorrhoea. Lancet 1: 1195, 1977.

2. PETTERSSON F., FRIES H., NILLIUS S.J.: Epidemiology of Secondary Amenorrhea. A.M. J. Obstet. Gynecol. 117: 80, 1983.

3. KEYE W.R., CHANG J., JAFFE R.: Prolactin Secreting Pituitary Adenomas in Women with Amenorrhea or Galactorrhea. Obstet. Gynecol. Survey 32: 723, 1977.

4. FRANKS S., JACOBS H.S., HULL G.R., STEELE S.J., NAVARRO J.D.N.: Management of Hiperprolactinaemic Amenorrhea. Brit J. Obst. Gynec. 84: 241, 1977.

5. DEL CORRAL F., ZUÑIGA J.E.: Amenorrea Ponencia Oficial. Rev. Col. Obst. y Ginec. 26: 97, 1975. 
6. VILLARREAL J.: Amenorrea - Diagnóstico - Tratamiento - Revisión Actualización. Universitas Médica. Vol. II-2: 175, 1965.

7. HERNANDEZ S., GELAYA J.A., VELEZ. VASQUEZ J.L.: Síndrome de Amenorrea y Galactorrea Consideraciones Clínicas. Ginec. Obstet. Méx. 41: 431, 1977.

ठ. NAKANO R., HASHIBA N., WASHIO M., TOJO S.: Diagnostic Evaluation of Progesterone. Acta Obstet. Gynecol. Scand. 58: 59, 1979.

9. YEN S.S.C., Chronic Anovulation. In Yen S.S.C., Jaffe R.B., (Eds). Reproductive Endocrinology. W.B. Saunder Company. Philadelphia, 1978, pp. 296-372.

10. SPEROFF L., GLASS R.H., KASE N.G. Amenorrea: Endocrinología Ginecológica y Esterilidad. The Williams and Wilkins Company. Baltimore, 1978.

11. DAVAJAN V. KLETZEY O.: Symposium on Adolescent Gynecology and Endocrinology. Pare 11: Secondary Amenorrhea, West \& Med. 131: 516, 1979.

12. ZAITZMAN M. da Z., ZAMORA A., CABALLERO M. de D., BAJERES M., RODRIGUEZ O.: Amenorrea en el Servicio de Ginecología del Hospital "José María Vargas" de Caracas. Rev. de Obst. y Gin. de Venezuela, 34: 337, 1976.
13. KLETZKY O.A., DAVAJAN Y., NAKAMURA R., MISHELL D.R.: Classification of Secondary Amenorrhea Based on Distinct Hormonal Patterns. J. Clin. Endocrinol. Metab. 41: 660, 1975.

14. SHEARMAN R.P., FRASER I.S.: Impact of New Diagnostic Methods on the Differential Diagnosis and Treatment of Secondary. Coment ed. Obstet. Gynecol. Survey. 32: 747.

15. COMBES R., JAQUET PH., GUIBOUT M., ANGELETTI S., TRAMONI M.: L'Exploration Hormonale Actuelle Des Amenorrhea S.J. Gynecol. Obstet. Biol. Reprod. 8: 27, 1979.

16. JACOBS H.S., HULL M.G.R., MURRAY M.A.F., FRANKS S.: Therapy Orentated Diagnosis of Secondary Amenorrhea. Hormone Res. 6: 268, 1975.

17. LUNENFELD B., BARZELATT J.,SPIELER J. Desing of Studes for the Assessment of Drugs and Hormones Used in the Treatment of Endocrine Forms of Female Infertility. In Diczfalusy (Eds). Regulation of Human Fertility. WhoSimposium Moscú, 1976. Bogtrykkiet Forum Copenhaeen. 1977, pp. 135-151. 